\title{
A WATER STATION MODEL WITH FUZZY C-MEANS ALGORITHM TO PROMOTE THE ABILITY OF WATER DISTRIBUTION SYSTEMS
}

\author{
Hong-Min Shau \\ Department of Harbor and River Engineering, National Taiwan Ocean University. Keelung, Taiwan 202, ROC \\ Wen-Chih Huang \\ Department of Harbor and River Engineering, National Taiwan Ocean University. Keelung, Taiwan 202, ROC, \\ huangwc@mail.ntou.edu.tw \\ Bi-Liang Lin \\ Department of Civil Engineering, MingHsin University of Science and Technology, Hsinchu, Taiwan 304, ROC \\ Jui-Min Hsiao \\ Department of Industrial Engineer and Management, Fortune Institute of Technolog
}

Follow this and additional works at: https://jmstt.ntou.edu.tw/journal

Part of the Civil and Environmental Engineering Commons

\section{Recommended Citation}

Shau, Hong-Min; Huang, Wen-Chih; Lin, Bi-Liang; and Hsiao, Jui-Min (2005) "A WATER STATION MODEL WITH FUZZY C-MEANS ALGORITHM TO PROMOTE THE ABILITY OF WATER DISTRIBUTION SYSTEMS," Journal of Marine Science and Technology. Vol. 13: Iss. 1, Article 4.

DOI: 10.51400/2709-6998.2101

Available at: https://jmstt.ntou.edu.tw/journal/vol13/iss1/4

This Research Article is brought to you for free and open access by Journal of Marine Science and Technology. It has been accepted for inclusion in Journal of Marine Science and Technology by an authorized editor of Journal of Marine Science and Technology. 


\title{
A WATER STATION MODEL WITH FUZZY C-MEANS ALGORITHM TO PROMOTE THE ABILITY OF WATER DISTRIBUTION SYSTEMS
}

\author{
Hong-Min Shau*, Wen-Chih Huang*, Bi-Liang Lin**, and Jui-Min, Hsiao***
}

Key words: water station model, water distribution system, district networks, fuzzy C-means algorithm.

\section{ABSTRACT}

A water station model will simulate the performance of a water distribution system for a given station location and quantity patterns in order to analyze present population distribution in the overall region, establish a reasonable local sample for each district network and obtain the optimal solution of the water supply stations. To establish a local sample, this model uses the Fuzzy C-Means algorithm (FCM algorithm) of the fuzzy clustering method. The sites have been modified with the mobile method so that the group points can be located at places nearest to the roadside in order to achieve the minimum cost. However, these points may not be the least costly solution. Capital benefit and efficiency are discussed in accordance with the local results obtained for each district. It is applicable to the group site for each water supply district to establish water supply stations of multi-functions.

\section{INTRODUCTION}

Aside from the problem of maintaining water supplies, which can satisfy basic human needs; the demand for water is increased in diversity, convenience, reliability and quality. Examples of this recent trend include the emergence of new products like purified water, ionized water, magnetized water and oxygenated water. Water supply stations that form part of the household water supply system can provide their local communities with high quality drinking water. Moreover, if the household water supply system is unable to supply water during civil emergencies, then back-up water sources can supply the water stations.

This analysis explores various of methods in ac-

Paper Submitted 06/30/04, Accepted 10/15/04. Author for Correspondence: Wen-Chih Huang. E-mail: huangwc@mail.ntou.edu.tw.

*Department of Harbor and River Engineering, National Taiwan Ocean University. Keelung, Taiwan 202, ROC.

**Department of Civil Engineering, MingHsin University of Science and Technology, Hsinchu, Taiwan 304, ROC.

***Department of Industrial Engineer and Management, Fortune Institute of Technology. cordance with the traditional clustering theories and fuzzy clustering theories of Ball \& Hall [1] and Zadeh. The methods of Roubens [8] and Kaufman \& Rousseruw [6] are also used, but have been altered to get the clusters' minimum degree of divergence. Other scholars who developed the fuzzy clustering algorithms include Jim [5], Roubens [9], Gath and Geva [4], Dubuisson [3], Xie and Gerardo [14]. Among their research, the FCM algorithm posed by Jim [5] is applied most extensively here.

This research aims to provide an easy and reasonable programming method that uses fuzzy clustering to replace traditional clustering. The approach presented here can help water supply companies seeking to enhance the effectiveness of established water supply stations and thus improve the quality of customer service. The water stations models for simulating water supply system, to establish a reasonable local sample for each district using the Fuzzy C-Means algorithm (FCM algorithm) of the fuzzy clustering analytic method, and to search for the necessary quantity and location of total water supply stations in the overall region with system simulation. Minimizing construction costs is taken as the objective function $\operatorname{Jm}(U, V)$, that can used to obtain the optimal solution of the water supply stations.

\section{THE WATER STATION MODEL}

The water station model for simulating water supply system performance is to establish a reasonable local sample for each district networks using the FCM algorithm. This method is described as follows:

$$
J_{m}(U, V)=\sum_{i=1}^{\kappa} \sum_{i=1}^{s}\left(u_{i j}\right)^{m} d^{2}\left(P_{j}, V_{i}\right)
$$

The objective function shown in equation (1) to represent the weighted time distant square from each data $P_{j}$ point to the center of each $V_{i}$ cluster; the weight value is equal to $\left(u_{i j}\right)^{m}$. Where $u_{i j}$ is the membership function value from the sample data point $j$ to the cluster $i$; $m$ is called "exponential weight." The bigger is $m$, the 
lesser is the influence of the smaller membership function value on the entire objective function. Therefore, the purpose of the exponential weight is to lower the effect caused by the noise when calculating the cluster's center value and the objective function value [12]. This research takes the objective function to be the reference of convergence or not for all kinds of possible cluster values to get the membership function value $u_{i j}$.

The FCM algorithm method presented in this article shows that can achieve an efficient supply of high quality drinking water regardless of the situation in now circumstance. This study covers significant issues visa-vis water supply distribution as follows:

1. Discussion of models for the construction of water supply stations mean to provide an optimal household water supply system.

2. Discussion of models for simulating the performance of water supply systems for each given station location and quantity patterns, this discussion will:

(1) Analyze present population distribution in the overall region.

(2) Establish a reasonable local sample for each district using the FCM algorithm from the fuzzy clustering analytic method.

(3) Outline the necessary quantity and appropriate location of all water supply stations in each given region using system simulation.

3. An analysis of the potential to minimize construction costs: Cost efficiency is taken as an ultimate objective. The FCM algorithm, which has proven to be an effective and reliable method for such an analysis, is used here to obtain the optimal solution for water supply stations.

4. Numerical example: Various water supply models are established in order to demonstrate how to offer the local community water of a consistently higher quality that can satisfy demand.

This model considers a different type [11], and the application of fuzzy clustering to locate the distribution locations of water supply stations is also outlined. The model here consists of three phases. In the first phase, the FCM algorithm is applied to find the initial clustering points. However, these points may not meet the actual requirements since they are not usually located alongside existing roads. Hence, in the second phase, an attempt is made to move these points to the nearest roads. The relocated points, though, may not provide the optimal solution at the lowest cost, As such, in the third phase, the cluster points generated in the first phase are re-used to identify feasible locations as close as possible to the roads. The genetic algorithms from the second phase are then used to find the least costly optimal solution. Finally, the solutions of the second and the third phases are compared.

\section{The FCM algorithm}

Before clustering the population in this area, the FCM algorithm must estimate the population distribution in the selected area of water supply station. This water station should be set in concentration of area. So we can use a single population distribution point to represent fifty or one hundred people, and official census information can be used as a reference to estimate the total population of the area. That can avoid population distribution to disorganize.

Water supply stations are usually to be set up alongside existing road, since they must be conveniently located. This study uses maps and information provided by the municipal government to outline the actual road distribution. The information is then defined by two linear equations. All roads are categorized as either ordinary roads, expressways, highways, tunnels, bridges and traffic circles. Since it is inappropriate to set up water supply stations near tunnels, bridges or traffic circles, field surveys must be conducted to determine feasible solutions. This work rejects unfeasible solutions in an attempt to minimize the range of feasible solutions. This can be defined by the linear equations.

Let the distribution points be defined as $(j=1,2$, $\ldots, s)$ where s represents total number of , the value of which is set according to the local population distribution. For example, if the local population is 5000 and the decision-maker uses one point to represent 100 people, then $s=5000 / 100=50$. From this, the computer can randomly select 50 points to show the local population distribution, defined as $\left(x_{j}^{p}, y_{j}^{p}\right)$.

The next step is to use fuzzy clustering to find optimum locations for the establishment of water supply stations. This study uses FCM algorithm to achieve this. The FCM algorithm assumes that the similarities between data points are measured by corresponding distance. If the distance is short between some data points and one of the cluster centers, then the data points can be said to be close to each other.

Using the FCM algorithm, s (i.e. the number of collected vectors $p_{j}$ ) is divided into $k$ fuzzy clusters, and the core of each cluster is determined according to the lowest cost or shortest distance identified by the objective function. When separating the various data points based on the clusters to which they belong, the membership grade is determined according to the membership rank, where the values of the membership grade $u_{i j}$ vary between 0 and 1 . The equation follows:

$$
u_{i j}=\frac{1}{\sum_{q=1}^{k}\left(\frac{d_{p i}}{d_{p q}}\right)^{2 / m-1}}=\frac{1}{\sum_{q=1}^{k}\left(\frac{\left\|p_{j}-v_{i}\right\|^{2 / m-1}}{\left\|p_{j}-v_{q}\right\|}\right)^{2 / 2}}
$$




$$
\begin{gathered}
=\frac{1}{\sum_{q=1}^{k}\left(\frac{\sqrt{\left(x_{j}^{p}-x_{i}^{v}\right)^{2}+\left(y_{j}^{p}-y_{i}^{v}\right)^{2}}}{\sqrt{\left(x_{j}^{p}-x_{q}^{v}\right)^{2}+\left(y_{j}^{p}-y_{q}^{v}\right)^{2}}}\right)} \\
=\frac{1}{\sum_{q=1}^{k}\left(\frac{\left(x_{j}^{p}-x_{i}^{v}\right)^{2}+\left(y_{j}^{p}-y_{i}^{v}\right)^{2}}{\left(x_{j}^{p}-x_{q}^{v}\right)^{2}+\left(y_{j}^{p}-y_{q}^{v}\right)^{2}}\right)} \\
q=1,2, \ldots, k ; i=1,2, \ldots, k ; j=1,2, \ldots, s ; p_{j} \in P
\end{gathered}
$$

where $P$ : the total number of distribution points set according to population distribution; $i$ : the water supply station, the cluster center of distribution points; $p_{j}$ : the distribution point $j$ set according to population distribution; $q$ : the water supply station that is similar to $i$ but another point; $n$ : the total number of roads in the area; $k$ : the total number of scheduled water supply stations in the area; $s$ : the total number of distribution points $p_{j}$ in the area set according to population distribution; $m$ : the weighted index determined by the decision-maker; $d_{p i}$ : the distance from distribution point $p_{j}$ to water supply station $i ; d_{p q}$ : the distance from distribution point $p_{j}$ to water supply station $q ; x_{i}^{v}$ : the coordinate $X$ of the cluster center $i$ after one fuzzy clustering; $y_{i}^{v}$ : the coordinate $Y$ of the cluster center of $i$ after one fuzzy clustering; $x_{i}^{p}$ : the coordinate $X$ of the distribution point $p_{j}$ set according to population distribution; $y_{i}^{p}$ : the coordinate $Y$ of the distribution point $p_{j}$ set according to population distribution.

\section{The water station model of the shortest distance}

Supposing the area has $\mathrm{n}$ roads, the road information can be represented as linear equations:

$$
\begin{aligned}
& a_{l} x+b_{l} y+c_{l}=0, l=1,2, \ldots, n, \\
& a_{l} \in R, b_{l} \in R, c_{l} \in R
\end{aligned}
$$

Based on the pre-assigned number of clusters $k$, the fuzzy clustering algorithm is used to generate $k$ cluster centers. The cluster centers namely represent by $w_{i}(i=1,2, \ldots, k)$. The corresponding coordinates of which are defined by $\left(x_{i}, y_{i}\right)$. However, the cluster centers generated by the fuzzy clustering algorithm might not be located beside roads. Since locations along roads are already considered to be feasible for water supply stations, the clustered points must be moved to locations along side roads. However, determining which roads the points should be moved to, and to which location along those roads, remains a problem, A feasible solution must therefore be found for the revised cluster point.

Moving the initial cluster center $w_{i}$ (generated by the fuzzy clustering algorithm) to the most suitable road requires considering the vertical distance from the generated cluster center to each road. When the vertical distance between the cluster centers and a given road is minimized, the road can be considered a feasible location for the movement. The equation (4) for calculating the vertical distance is as follows:

$$
\begin{aligned}
& d_{i l}=\frac{a_{l} x_{i}+b_{l} y_{i}+c_{l}}{\sqrt{a_{l}^{2}+b_{l}^{2}}} \\
& l=1,2, \ldots, n ; i=1,2, \ldots, k
\end{aligned}
$$

where $a_{l} x+b_{l} y+c_{l}=0$ denotes the equation of road and the corresponding coordinates of cluster center $i$ are $\left(x_{i}\right.$, $\left.y_{i}\right)$. Finally, the new coordinates of cluster center $i$ are defined by $\left(x_{i}^{\text {new }}, y_{i}^{\text {new }}\right)$. The revised feasible water supply station is then allowed to be $w_{i}$. The minimum cost function of the objective function, using FCM algorithm is then stated as:

$$
\operatorname{Min} J_{m}(U, V)=\sum_{i=1}^{\kappa} J_{i}=\sum_{i=1}^{\kappa} \sum_{j=1}^{s}\left(u_{i j}\right)^{m} d_{i j}^{2}
$$

where $u_{i j}$ denotes the membership grade (which varies between 0 and 1 ) and $w_{i}$ is the revised cluster center $i$; or the new location of the water supply station on road $w_{i}$ with the coordinates $\left(x_{i}^{\text {new }}, y_{i}^{\text {new }}\right)$.

The Euclidean distance from the new cluster center $i$ can be found by equations (6):

$$
d_{i j}=\left\|w_{i}-p_{i}\right\|, j=1,2, \ldots, s
$$

where $d_{i j}$ represents the Euclidean distance from the new cluster center $i$ to data point $j$.

That will allow the calculation of the cost function of the FCM algorithm as equations (7):

$$
\begin{aligned}
\operatorname{Min} J_{m}(U, V) & =\sum_{i=1}^{\kappa} J_{i}=\sum_{i=1}^{\kappa} \sum_{j=1}^{s}\left(u_{i j}\right)^{m}\left[\left(x_{i}^{\text {new }}-x_{j}^{p}\right)\right. \\
& \left.+\left(y_{i}^{\text {new }}-y_{j}^{p}\right)^{2}\right]
\end{aligned}
$$

where $x_{i}^{\text {new }}$ denotes the new coordinate $X$ of cluster center $i ; y_{i}^{\text {new }}$ denotes the new coordinate $Y$ of cluster center $i$.

\section{The revised model of water supply stations alongside roads}

The area is meant to have $\mathrm{n}$ roads, the road information which can be represented by equation (3). The fuzzy clustering algorithm is used to generate $k$ cluster 
points. However, the generated points may not happen to be alongside any road; that is, when the coordinates in any equations regarding roads are used, $a_{l} x+b_{l} y+c_{l}$ may not equal 0 .

Since it is considered feasible to place water supply stations alongside roads, this is where the clustered points are relocated. Difficulties may then arise regarding which roads the points should be moved to, and which point is feasible while minimizing the cost function. Consequently, the theory presented here assumes that the relocated new water supply station is $w_{i}$ $(i=1,2, \ldots, k)$. The target equation (5) should be modified.

The new cluster center i represented as $w_{i}$, this is the location of the relocated water supply station. This refers to a person, Its coordinates are $\left(x_{i}^{\text {new }}, y_{i}^{\text {new }}\right), d_{i j}=$ $\left\|w_{i}-p_{i}\right\|,(i=1,2, \ldots, k ; j=1,2, \ldots, s)$. The next task becomes moving the initial cluster centers , to the road side. To do this, the points generated by fuzzy clustering and the road distribution is used to identify surrounding roads that the cluster can reasonably be moved to. The roads may meet the requirements and be used to determine the position of the points. If the information is outlined using mathematical programming, the number of roads to which the cluster may be moved to must be determined, and the vertical distance from the points to each road must be calculated. The distances must then be sequenced, and $G$ number of roads with the smallest vertical distance (according to the predetermined number $G$ ) can be selected as the feasible solution. The equation for calculating the vertical distance is shown by equation (3).

The corresponding coordinates of cluster center $i$ are $\left(x_{i}, y_{i}\right)$. After sequencing the roads according to the vertical distance $d_{i l}$, the equation for roads near the initial point $i$ is identified. These road equations are then used as constraints for mathematical programming. This study then assumes that the sequential road equations will vary according to different cluster centers and the following general equation can be applied:

$$
\begin{aligned}
& a_{i r} x+b_{i r} y+c_{i r}=0, r=1,2, \ldots, n, \\
& i=1,2, \ldots, k, a_{i r} \in R, b_{i r} \in R, c_{i r} \in R
\end{aligned}
$$

This calculation shows the road $r$ equation after the initial cluster center $i$ is sequenced according to the distance. However, only the first $G_{i}$ road equations represent feasible solutions for the movement of the cluster center $i$. The location of $G_{i}$ is determined by road distribution. If the point is on the line, then the equation is 0 . Hence, to have points located on the first $G$ road equations, the product of these equations must be 0 ; thus the feasible solutions located on these roads will have a product of 0 . The feasible coordinates of cluster center $i\left(x_{i}^{\text {new }}, y_{i}^{\text {new }}\right)$ can be obtained when $i=1,2, \ldots, k$ and the substitution of the coordinates into the equation achieves a result of 0 . The procedure is denoted as equations (9):

$$
\begin{aligned}
& \left(a_{i 1} x_{i}^{\text {new }}+b_{i 1} y_{i}^{\text {new }}+c_{i 1}\right) \times\left(a_{i 2} x_{i}^{\text {new }}+b_{i 2} y_{i}^{\text {new }}+c_{i 2}\right) \\
& \times \cdots \times\left(a_{i r} x_{i}^{\text {new }}+b_{i r} y_{i}^{\text {new }}+c_{i r}\right)=0
\end{aligned}
$$

After the following arrangement as equations (10):

$$
\prod_{r=1}^{G i}\left(a_{i r} x_{i}^{\text {new }}+b_{i r} y_{i}^{\text {new }}+c_{i r}\right)=0
$$

The original target equation thus becomes as equations (11):

$$
\begin{aligned}
\operatorname{Min} J_{m}(U, V) & =\sum_{i=1}^{\kappa} J_{i}=\sum_{i=1}^{\kappa} \sum_{j=1}^{s} u_{i j}^{m}\left[\left(x_{i}^{\text {new }}-x_{j}^{p}\right)\right. \\
& \left.+\left(y_{i}^{n e w}-y_{j}^{p}\right)^{2}\right]
\end{aligned}
$$

s.t.

$$
\begin{aligned}
& \prod_{r=1}^{G_{i}}\left(a_{i r} x_{i}^{\text {new }}+b_{i r} y_{i}^{\text {new }}+c_{i r}\right)=0, \\
& i=1,2, \ldots, k, r=1,2, \ldots, G_{i} \\
& \sum_{i=1}^{k} u_{i j}=1, a_{i r} \in R, b_{i r} \in R, c_{i r} \in R, \\
& j=1,2, \ldots, s, m \in[1, \infty)
\end{aligned}
$$

Where $G_{i}$ : the number of surrounding roads close to cluster center i after sequencing; $a_{i r}$ : the parameters of road equation of road $r$ around cluster center $i$ after they are put in sequence; $b_{i r}$ : the parameters of road equation of road $r$ around cluster center $i$ after they are put in sequence; $c_{i r}$ : the parameters of road equation of road $r$ around cluster center $i$ after they are put in sequence; $u_{i j}$ : varies between 0 and 1 , which denotes the membership grade of the distribution point $p_{j}$ from the set $j$ to the new cluster center $i$. Using the equation (2):

$$
u_{i j}=\frac{1}{\sum_{q=1}^{k}\left(\frac{\left(x_{j}^{p}-x_{i}^{n e w}\right)^{2}+\left(y_{j}^{p}-y_{i}^{n e w}\right)^{2}}{\left(x_{j}^{p}-x_{q}^{n e w}\right)^{2}+\left(y_{j}^{p}-y_{q}^{n e w}\right)^{2}}\right)^{1 / m-1}}
$$

\section{NUMERCIAL EXAMPLE}

This section presents a numerical case study, including testing and analysis. First, the XX section of Tainan City (an area of 1000 hectares with G roads from Tainan City Government, as shown in Figure 1) has 
been selected as the test example. The Matlab software has been adapted for the purposes of this simulation. As described in section 2 , the solution solving comprises several phases.

\section{The generation of the population distribution points}

The population distribution points are generated randomly with the help of the "random" function in the Matlab software. Each distribution point represents 100 people according specification of a manufactured item of water station. From the description in section 2, we can predict that the population in the selected area to be approximately 5000 . Therefore, 50 population distribution points must be randomly generated. Table 1 illustrates the coordinates of the simulated population distribution points generated by the Matlab. Figure 2 shows their relative positions on the map.

Figure 2 shows the randomly generated population distribution point. Here, $X$ and $Y$ represent the coordi-

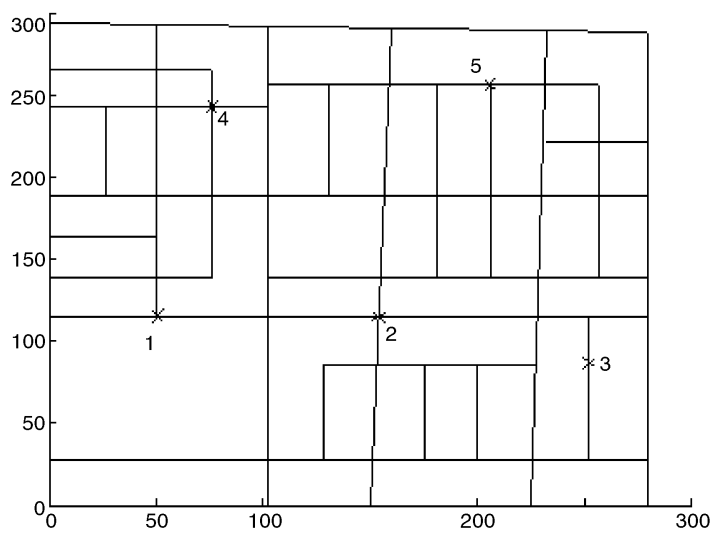

Fig. 1. XX section of Tainan City with $\mathrm{G}$ roads and 5 traditional water stations.

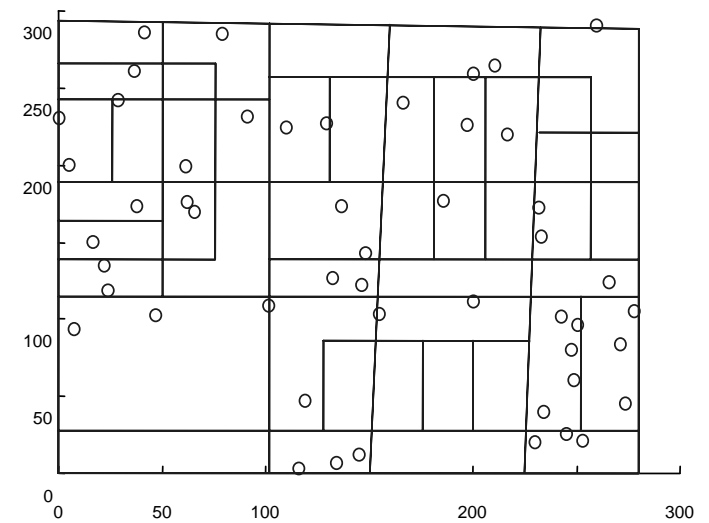

Fig. 2. The coordinates of 50 population distribution points.
Table 1. The coordiantes and the clustering results of numercial example

\begin{tabular}{|c|c|c|c|}
\hline No. & $X$ & $\mathrm{Y}$ & Groups \\
\hline 1 & 129.11 & 227.90 & 4 \\
\hline 2 & 91.00 & 232.36 & 4 \\
\hline 3 & 4.78 & 200.87 & 4 \\
\hline 4 & 197.24 & 226.74 & 5 \\
\hline 5 & 265.75 & 124.34 & 3 \\
\hline 6 & 252.58 & 21.07 & 3 \\
\hline 7 & 78.63 & 285.39 & 4 \\
\hline 8 & 21.63 & 135.33 & 1 \\
\hline 9 & 65.70 & 169.88 & 1 \\
\hline 10 & 100.93 & 109.41 & 2 \\
\hline 11 & 232.83 & 153.60 & 5 \\
\hline 12 & 41.26 & 286.50 & 4 \\
\hline 13 & 250.31 & 97.09 & 3 \\
\hline 14 & 61.84 & 176.20 & 1 \\
\hline 15 & 145.82 & 122.92 & 2 \\
\hline 16 & 248.38 & 60.68 & 3 \\
\hline 17 & 185.73 & 177.25 & 5 \\
\hline 18 & 37.31 & 174.10 & 1 \\
\hline 19 & 6.97 & 94.42 & 1 \\
\hline 20 & 210.57 & 265.46 & 5 \\
\hline 21 & 16.40 & 150.06 & 1 \\
\hline 22 & 23.80 & 118.69 & 1 \\
\hline 23 & 271.12 & 84.33 & 3 \\
\hline 24 & 147.69 & 143.04 & 2 \\
\hline 25 & 0.03 & 231.24 & 4 \\
\hline 26 & 273.04 & 45.95 & 3 \\
\hline 27 & 200.09 & 259.70 & 5 \\
\hline 28 & 46.89 & 102.89 & 1 \\
\hline 29 & 277.77 & 105.89 & 3 \\
\hline 30 & 36.15 & 261.77 & 4 \\
\hline 31 & 200.24 & 111.57 & 2 \\
\hline 32 & 136.07 & 173.34 & 2 \\
\hline 33 & 28.59 & 242.54 & 4 \\
\hline 34 & 166.16 & 240.75 & 5 \\
\hline 35 & 216.62 & 220.33 & 5 \\
\hline 36 & 115.87 & 3.84 & 2 \\
\hline 37 & 231.64 & 173.20 & 5 \\
\hline 38 & 233.87 & 40.24 & 3 \\
\hline 39 & 144.78 & 12.49 & 2 \\
\hline 40 & 118.74 & 47.53 & 2 \\
\hline 41 & 132.06 & 127.16 & 2 \\
\hline 42 & 154.60 & 103.51 & 2 \\
\hline 43 & 133.89 & 7.12 & 2 \\
\hline 44 & 259.46 & 290.62 & 5 \\
\hline 45 & 242.13 & 102.02 & 3 \\
\hline 46 & 244.57 & 26.12 & 3 \\
\hline 47 & 246.97 & 80.56 & 3 \\
\hline 48 & 109.74 & 224.35 & 4 \\
\hline 49 & 229.48 & 20.95 & 3 \\
\hline 50 & 61.51 & 199.64 & 4 \\
\hline
\end{tabular}


nates on the map of the planned area. Note that the 50 population distribution points are divided into five groups in this example. Table 1 shows the clustering results.

\section{The using of the FCM algorithm}

The FCM algorithm of the fuzzy clustering method is used for clustering. According to the description in previous section, the FCM algorithm is used to determine the core point of each cluster based on the lowest cost or shortest distance found by the objective function. When separating the data points into individual clusters, a degree of fitness is assigned according to the level of fitness. The value of degree of fitness varies between 0 and 1 . The general definition of the objective function of the FCM algorithm is shown as equation (5). Thus, the following steps are used to find the optimal solution with FCM algorithm.

Step 1: Use a random number to set the initial membership matrix $U$, and normalize $U$ to ensure that the values in the matrix vary between 0 and 1 to satisfy as equation (15).

$$
J_{m}(U, V)=\sum_{i=1}^{\kappa} \sum_{j}^{s} u_{i j}^{m} d_{i j}^{2}+\sum_{j=1}^{s} \lambda_{j}\left(\sum_{i=1}^{\kappa} u_{i j}-1\right)
$$

where $\sum_{i=1}^{k} u_{i j}=1, \lambda_{j}$ is the Lagrange multiplier in each constrains.

Step 2: Use the FCM algorithm to calculate the fuzzy cluster center. $w_{i}(i=1,2, \ldots, k)$.

Step 3: Use equation (5) to calculate the objection function. The calculation should stop if: (1) the solved value is below a certain tolerance value; (2) the corrected value is below a certain threshold value; or (3) the number of steps exceeds a certain value.

Step 4: Use $w_{i}$ to determine the new membership matrix $U$. After repeated calculations as shown above, the fuzzy cluster center can be obtained.

The Table 2 has listed five traditional stations (a spot shown in Figure 1) by experience and the clustering results. We can solving the solution according as the equation (7). $J_{m}(U, V)=90285.2$

The program was coded for these steps, and a subprogram built into the fuzzy toolbox of the Matlab in order to simulate the clusters and cluster centers of the FCM. Table 2 lists the clustering results and the cluster. Figure 3 also shows the corresponding geographic positions of the coordinates. After implementing the FCM 100 times, the value of the target equation was revised to reach a final value of 71130.9 (from an initial value of 189105.5).

\section{The revised solution alongside the road}

As previously discussed, water supply stations should be established alongside the road. These results show that some cluster centers do not satisfy this requirement. Hence, the results of the fuzzy clustering must be clarified. In attempt to correct the results in this example, the cluster centers, which were not located alongside the road, are used to determine the roads that are closest to the clusters by calculating the Euclidean distance. The vertical distance between the cluster centers and the roads is also calculated. The roads with the shortest vertical distances to the cluster centers are considered feasible solutions for the target spot. The equation for calculating the vertical distance is as equation (4).

Consequently, the cost function is revised to equation (11). The revised target spot is depicted by "** in Figure 4. Table 3 lists the result of the relocated center. The new objective value is 71131.0 , which is convenient to people to take water from water station. The result is not significantly different from the value 71130.9 before revised.

Table 2. The traditional \& fuzzy clustering results

\begin{tabular}{ccrrr}
\hline center & \multicolumn{2}{c}{ Traditional center } & \multicolumn{2}{c}{ Fuzzy cluster } \\
& \multicolumn{1}{c}{ X } & \multicolumn{1}{c}{ Y } & \multicolumn{1}{c}{ X } & \multicolumn{1}{c}{ Y } \\
\hline 1 & 34.04 & 136.59 & 49.50 & 139.00 \\
2 & 143.21 & 107.79 & 151.50 & 115.20 \\
3 & 248.15 & 66.42 & 252.00 & 80.60 \\
4 & 49.79 & 245.05 & 211.90 & 245.10 \\
5 & 201.80 & 234.99 & 201.90 & 234.99 \\
\hline
\end{tabular}

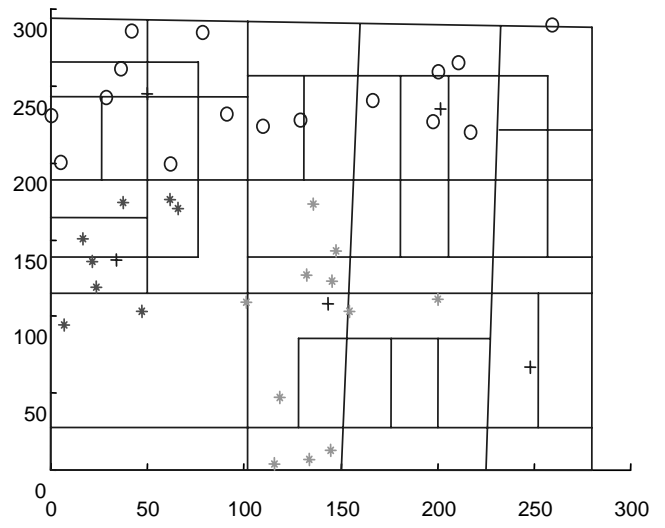

Fig. 3. The corresponding positions of the relocated center. 
Table 3. The result of the relocated center to the road side

\begin{tabular}{crr}
\hline relocated center & \multicolumn{1}{c}{ X } & \multicolumn{1}{c}{ Y } \\
\hline 1 & 34.04 & 139.00 \\
2 & 143.21 & 115.00 \\
3 & 252.00 & 66.42 \\
4 & 50.00 & 245.05 \\
5 & 206.00 & 234.99
\end{tabular}

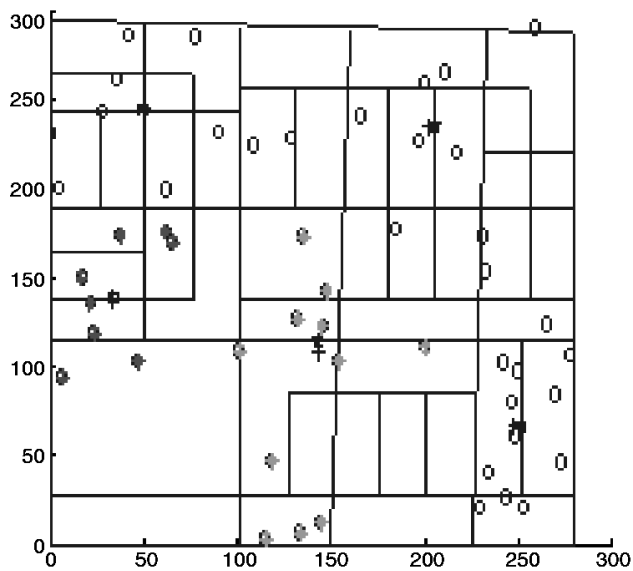

Fig. 4. The revised target spot alongside the road.

\section{CONCLUSION}

This study aims to provide an easy and reasonable programming method that uses the fuzzy C-means clustering (FCM) algorithm to replace traditional clustering. The approach presented here can help water supply companies seeking to enhance the effectiveness of established water supply stations, and thus improve the quality of customer service.

Users of water supply stations usually live close to the stations that they use. However, a minority of users may live a considerable distance from their relative water supply station. The new approach presented here achieves more flexible results than traditional clustering. This study maintains the assumption that most water station users live close to their stations, but also considers situations that involve uncertainty and fuzziness. Consequently, compared with other methods or models, fuzzy theory shows much more flexibility in solving to set the optimal station and location.

This is the first study to attempt to apply fuzzy clustering when setting up water supply stations and it avoids the weaknesses of traditional methods such as by experience to set the station. This work thus provides water supply companies with a new approach for optimizing the placement of water supply stations. This approach can also be applied to drinking water system construction, and the establishment of chain stores or company branches. However, this study focuses on application, and does not compare the relative advantages of different approaches. For example, aside from the constraints of cost and distance considered in this approach, the model may also need to consider other practical difficulties.

\section{REFERENCES}

1. Ball, G. and Hall, D., Background Information on Clustering Techniques, Stanford Research Institute, CA (1968).

2. Bezdek, J.C., "Cluster Validity with Fuzzy Sets," J. Cybernetics, Vol. 4, pp. 95-104 (1974).

3. Dubuisson, G.T., "Similarity of Classes and Fuzzy Clustering, “Fuzzy Sets Syst., Vol. 34, pp. 213-221 (1990).

4. Gath, I. and Geva, A.B., "Unsupervised optimal fuzzy clustering," IEEE T. Pattern Anal., Vol. 11, No. 7, pp. 773-781 (1989).

5. Jim, C.B., Pattern Recognition with Fuzzy Objective Function Algorithms, Plenum Press, New York (1981).

6. Kaufman, L. and Rousseruw, P.J., An Introduction to Cluster Analysis, John Wiley and Sons Inc, CA (1989).

7. Punj, G. and Stewart, D.W., "Cluster Analysis in Marketing Research, Review and Suggestions for Application," J. Marketing Res., Vol. 20 (May), pp.134148 (1983).

8. Roubens, M., "Pattern Classification Problems and Fuzzy Sets," Fuzzy Sets Syst., Vol. 1, pp. 239-253 (1978).

9. Roubens, M., "Fuzzy Clustering Algorithms and their Cluster Validity," Eur. J. Oper. Res., Vol. 10, pp. 294-301 (1982).

10. Sugeno, M. and Yasukawa, T., "A Fuzzy-logic-based Approach to Qualitative Modeling," IEEE T. Fuzzy Syst., Vol. 1, No. 1, 7-31 (1993).

11. Tsai, Y.T. and Wang, K.S., "The Development of Modular-based Design in Considering Technology Complexity," Eur. J. Oper. Res., Vol.119, pp.692-703 (1999).

12. Windham, M.P., "Cluster Validity for the Fuzzy C-Means Clustering Algorithm," IEEE T. Pattern Anal., Vol. 4, No. 4, pp. 357-363 (1982).

13. Yager, R.R. and Filev, D.P., "Generation of Fuzzy Rules by Mountain Clustering," J. Intell. Fuzzy Syst., Vol., pp. 209-219 (1994).

14. Xie, X.L. and Gerardo, B., "A Validity Measure for Fuzzy Clustering,” IEEE T. Pattern Anal., Vol.13, No.8, pp. 841-847 (1991). 